\title{
The complete mitochondrial DNA genome of an unknown hominin from southern Siberia
}

\author{
Johannes Krause ${ }^{1}$, Qiaomei Fu ${ }^{1}$, Jeffrey M. Good ${ }^{2}$, Bence Viola ${ }^{1,3}$, Michael V. Shunkov ${ }^{4}$, Anatoli P. Derevianko ${ }^{4}$ \\ $\&$ Svante Pääbo ${ }^{1}$
}

With the exception of Neanderthals, from which DNA sequences of numerous individuals have now been determined ${ }^{1}$, the number and genetic relationships of other hominin lineages are largely unknown. Here we report a complete mitochondrial (mt) DNA sequence retrieved from a bone excavated in 2008 in Denisova Cave in the Altai Mountains in southern Siberia. It represents a hitherto unknown type of hominin mtDNA that shares a common ancestor with anatomically modern human and Neanderthal mtDNAs about 1.0 million years ago. This indicates that it derives from a hominin migration out of Africa distinct from that of the ancestors of Neanderthals and of modern humans. The stratigraphy of the cave where the bone was found suggests that the Denisova hominin lived close in time and space with Neanderthals as well as with modern humans ${ }^{2-4}$.

The first hominin group to leave Africa was Homo erectus about 1.9 million years $(\mathrm{Myr})$ ago $^{5}$. Archaeological as well as genetic data indicate that at least two groups of hominins left Africa after this event: first, the ancestors of the Neanderthals between 500,000 and 300,000 years ago (500 and $300 \mathrm{kyr}$ ago, respectively), presumably Homo heidelbergensis or Homo rhodesiensis ${ }^{6-9}$; and, second, anatomically modern humans about $50 \mathrm{kyr}$ ago ${ }^{10,11}$. Despite recent advances in the retrieval of ancient DNA from Neanderthals and early modern humans $^{12-14}$, DNA sequences have not been recovered from other Pleistocene hominins such as $H$. erectus, $H$. heidelbergensis or Homo antecessor ${ }^{15}$. A major reason for this is that exceptional circumstances are required for DNA to survive over long time periods. DNA degradation increases with temperature and soil conditions such as acidity $^{16}$, and most early hominin fossils come from equatorial and tropical regions in Africa and Eurasia, where conditions for DNA survival are therefore poor. Thus, although direct descendants of $H$. erectus might have survived until less than $100 \mathrm{kyr}$ ago in Indonesia $^{17}$, it is unlikely that endogenous DNA will be retrieved from these hominins. However, archaeological evidence suggests that archaic hominins such as $H$. erectus, $H$. heidelbergensis and Neanderthals also lived at higher latitudes where the potential for DNA preservation is better. One such region is the Altai Mountains in southern Siberia ${ }^{4}$ where hominin occupation may go back to more than $125 \mathrm{kyr}$ ago (for example, the Karama site ${ }^{18}$.

Fossils complete enough for morphological classification do not exist from most sites in the Altai. Rather, small pieces of human skeletons such as teeth and bone fragments are generally recovered ${ }^{4,19}$. In 2008, the distal manual phalanx of the fifth digit of a hominin was excavated in Denisova Cave $\left(51^{\circ} 40^{\prime} \mathrm{N} ; 84^{\circ} 68^{\prime} \mathrm{E}\right)$ in the Altai Mountains, Russia. Episodic hominin occupation has been documented at this site for at least $125 \mathrm{kyr}^{20}$ and the phalanx was found in a stratum (layer 11) dated to 48-30 kyr ago where an assemblage containing both Upper and Middle Palaeolithic elements has been reported (see Supplementary Information).

We extracted DNA from $30 \mathrm{mg}$ of bone powder and converted it into an Illumina sequencing library using DNA adaptors that carry project-specific barcodes. We next used a recently published protocol for targeted sequence retrieval called primer extension capture (PEC) ${ }^{1}$ to isolate mtDNA fragments from the entire mitochondrial genome. The isolated fragments were sequenced from both ends on the Illumina $\mathrm{GA}_{\text {II }}$ platform, using 76 cycles for each read. Fragments were included in further analyses if their forward and reverse reads overlapped by at least 11 base pairs (bp) and thus could be merged into single sequences. This removes all fragments over $\sim 134$ bp from analysis, but reduces errors at the $3^{\prime}$ ends of Illumina reads where error rates are highest ${ }^{21,22}$. We generated 1,178,300 merged sequences, of which 93,349 (7.9\%) aligned to the revised Cambridge Reference Sequence (rCRS) ${ }^{23}$ using an iterative mapping assembler, MIA ${ }^{1}$. This alignment program is particularly suitable for aligning ancient DNA sequences because it takes into account the frequent sequence errors associated with base damage in ancient DNA sequences ${ }^{24,25}$. Owing to the library amplification steps involved in the primer extension capture procedure, multiple copies of each original DNA molecule may be sequenced. Thus, fragments with identical start and end coordinates were merged to single sequences where at each position the base with the highest quality score was used. A total of 30,443 such distinct sequences were then used to assemble a mtDNA sequence ${ }^{1}$. The final assembled mtDNA sequence was identical irrespective of whether the reference mtDNA sequence used for the assembly was modern human or Neanderthal. Coverage across the mtDNA was high (mean $=156$-fold, lowest 2 -fold, highest 602-fold; Supplementary Fig. 1).

Several different approaches were used to assess the reliability of the mtDNA sequence determined. First, we made a second DNA extract from the Denisova phalanx and determined 9,908 mtDNA fragments by shotgun sequencing on the Illumina platform. From the reads we assembled a complete mtDNA sequence that was identical to the one retrieved with the PEC approach (Supplementary Information). This shows that the mtDNA sequence retrieved is reproducible and not dependent on the particular primers used for PEC. Second, we used 276 sequence positions where the Denisova hominin mtDNA is different from $>99 \%$ of present-day human mtDNAs to assess whether the mtDNA recovered with PEC comes from a single individual. Among the 15,008 fragments that overlap these 276 positions, 14,961 carry the bases of the Denisova hominin mtDNA. This suggests that $99.7 \%$ (95\% confidence interval; 99.6-99.8\%) of all mtDNA fragments in the bone come from a single individual. Third, we used three physical features of the DNA fragments sequenced to gauge their extent and patterns of degradation: (1) the average length of the

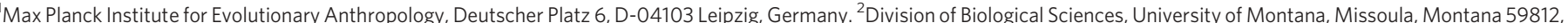

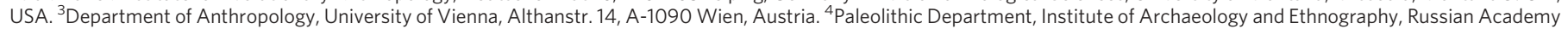
of Sciences, Siberian Branch, Lavrentieva Avenue, 17 Novosibirsk, RU-630090, Russia. 

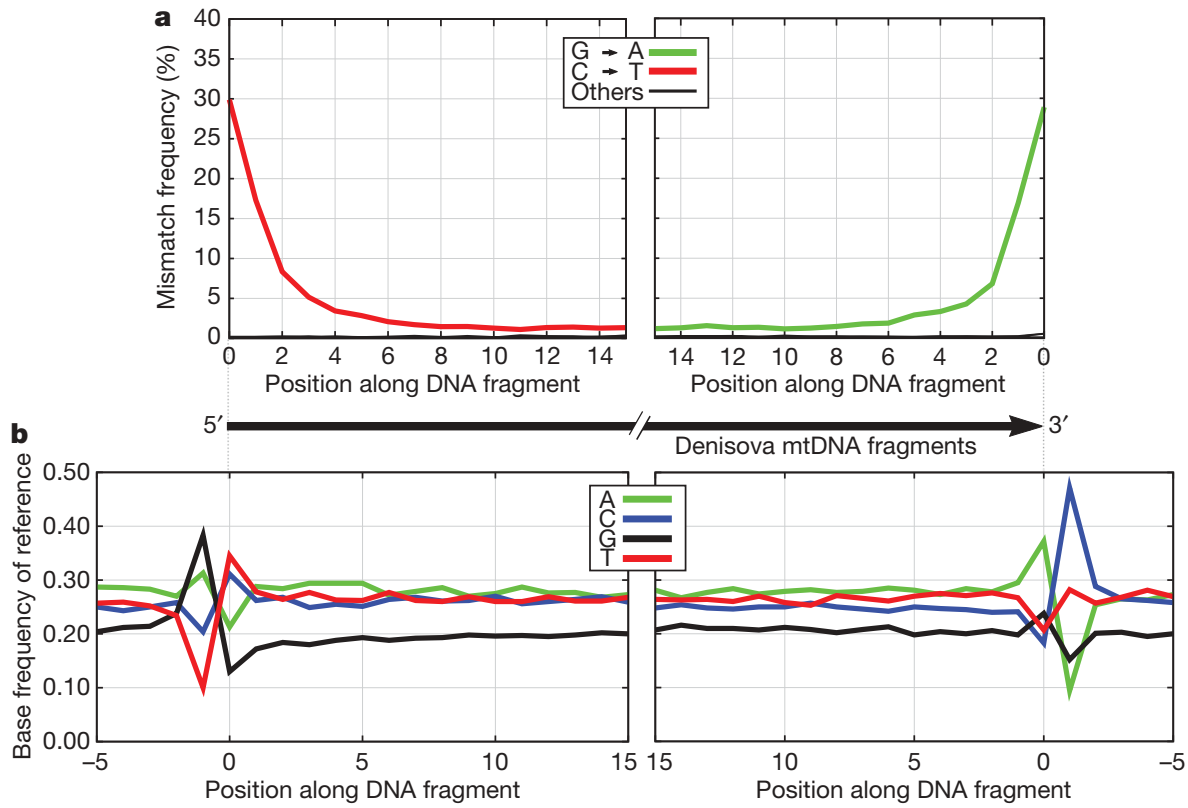

Figure 1 Physical features of Denisova mtDNA. a, b, Nucleotide misincorporation patterns (a) and nucleotide frequencies of the aligned reference sequence (b) at and around the ends of mtDNA fragments sequenced from the Denisova hominin.

fragments is $85.3 \mathrm{bp}$ (Supplementary Fig. 3); (2) at the $5^{\prime}$ ends of the fragments about $30 \%$ of cytosine residues are replaced by thymine residues whereas at the $3^{\prime}$ ends the complementary replacement of guanine residues by adenine residues is seen; (3) at the positions immediately outside the fragments purines (A and G) are overrepresented (Fig. 1). These three features have recently been shown to be typical of ancient DNA relative to contaminating DNA fragments in several Neanderthal specimens ${ }^{13}$. Because the mtDNA retrieved from the Denisova hominin stems overwhelmingly from a single individual and its extent and patterns of degradation as well as nucleotide misincorporations are typical of ancient DNA, the assembled mtDNA is likely to represent the mtDNA sequence carried by the Denisova individual when alive.

We aligned the Denisova hominin mtDNA sequence to 54 presentday modern human mtDNAs, a Late Pleistocene mtDNA recently determined from an early modern human from Kostenki, Russia ${ }^{13}$, six complete Neanderthal mtDNAs ${ }^{1,26}$, one bonobo (Pan paniscus) mtDNA and one chimpanzee (Pan troglodytes) mtDNA. Whereas Neanderthals differ from modern humans at an average of 202 nucleotide positions, the Denisova individual differs at an average of 385 positions (Fig. 2), and the chimpanzee at 1,462 positions (Supplementary Information). The Denisova hominin mtDNA thus

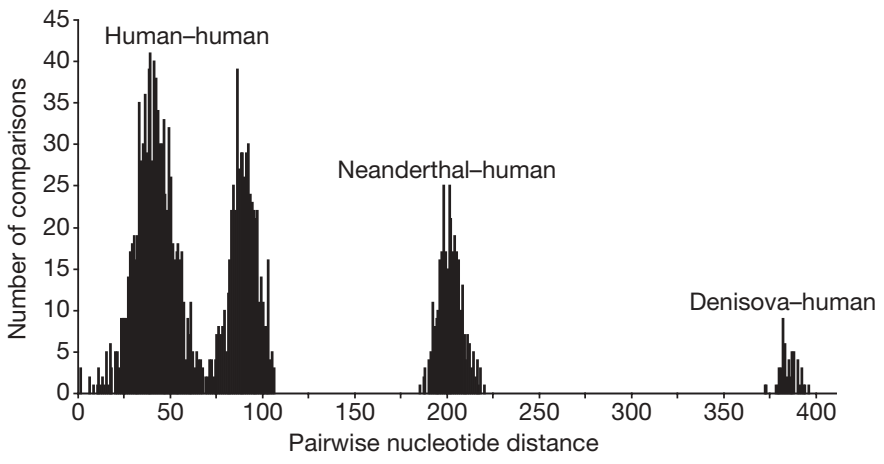

Figure 2 | Distribution of pairwise nucleotide differences. Pairwise nucleotide differences from all pairs of complete mtDNAs from 54 presentday and one Pleistocene modern human, six Neanderthals and the Denisova hominin are shown. carries almost twice as many differences to the mtDNA of presentday humans as do Neanderthal mtDNAs. A phylogenetic analysis similarly shows that the Denisova hominin mtDNA lineage branches off well before the modern human and Neanderthal lineages (Fig. 3). Assuming an average divergence of human and chimpanzee mtDNAs of 6 million years ago, the date of the most recent common mtDNA ancestor shared by the Denisova hominin, Neanderthals and modern humans is approximately one million years ago ( mean $=1,040,900$ years ago; 779,300-1,313,500 years ago, 95\% highest posterior density (HPD)), or twice as deep as the most recent common mtDNA ancestor of modern humans and Neanderthals ( mean $=465,700$ years ago; 321,200-618,000 years ago, 95\% HPD) (Fig. 3). Although the absolute dates depend on several assumptions and are subject to uncertainty (Supplementary Information), the fact that the divergence of the Denisova hominin mtDNA is about twice as old as the divergence of Neanderthal and modern human mtDNAs is robust to most assumptions.

The 12 proteins encoded by the Denisova hominin mtDNA (excluding ND6, Supplementary Information) show low per-site rates of amino acid replacements $\left(d_{\mathrm{N}}\right)$ when compared to the persite rates of silent substitutions $\left(d_{\mathrm{S}}\right)$, consistent with strong purifying selection influencing the evolution of the mitochondrial proteins $\left(d_{\mathrm{N}} / d_{\mathrm{S}}=0.056\right)$. Notably, when the evolution of mitochondrial protein-coding genes in modern humans, Neanderthals, chimpanzees and bonobos is gauged in conjunction with the Denisova hominin mtDNA, a previously described reduction of silent substitutions causing an increased $d_{N} / d_{S}$ in Neanderthals ${ }^{1,26}$ is not observed. This is probably due to a more accurate reconstruction of substitutional events when the long evolutionary lineage leading to modern humans and Neanderthals is subdivided by the Denisova hominin mtDNA (see Supplementary Information).

Although nuclear DNA sequences are needed to clarify definitively the relationship of the Denisova individual to present-day humans and Neanderthals, the divergence of the Denisova mtDNA lineage on the order of one million years shows that it was distinct from the initial radiation of $H$. erectus that first left Africa $1.9 \mathrm{Myr}$ ago, and perhaps also from the taxon $H$. heidelbergensis, if the latter is the direct ancestor of Neanderthals ${ }^{27}$. An unambiguous association of the Denisova mtDNA with morphologically defined hominin taxa awaits determination of mtDNA sequences from more complete skeletal remains. 


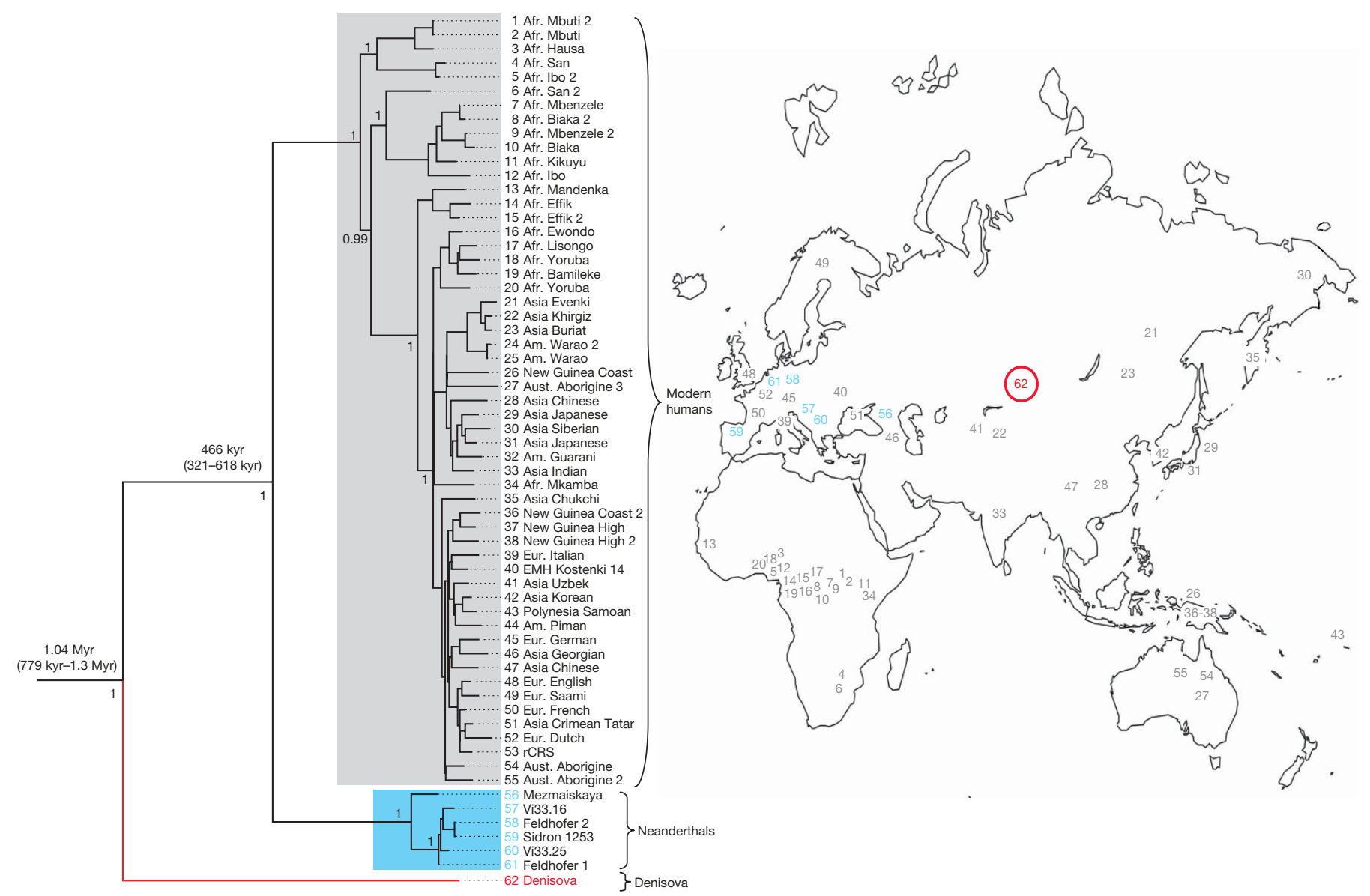

Figure 3 | Phylogenetic tree of complete mtDNAs. The phylogeny was estimated with a Bayesian approach under a GTR $+\mathrm{I}+\Gamma$ model using 54 present-day and one Pleistocene modern human mtDNA (grey), 6 Neanderthals (blue) and the Denisova hominin (red). The tree is rooted with a chimpanzee and a bonobo mtDNA. Posterior probabilities are given for

We note that the stratigraphy and indirect dates indicate that this individual lived between 30,000 and 50,000 years ago ${ }^{20,28}$. At a similar time individuals carrying Neanderthal $\mathrm{mtDNA}^{4}$ were present less than $100 \mathrm{~km}$ away from Denisova Cave in the Altai Mountains, whereas the presence of an Upper Palaeolithic industry at some sites, such as Kara-Bom and Denisova, has been taken as evidence for the appearance of anatomically modern humans in the Altai before 40,000 years ago $^{2,3}$. Although these dates are associated with large and unknown errors, this temporal concurrence suggests that complete and successive replacements of distinct hominin forms, similar to what occurred in Western Europe ${ }^{11}$, may not have taken place in southern Siberia. Rather, representatives of three genetically distinct hominin lineages may all have been present in this region at about the same time. Thus, the presence of Homo floresiensis in Indonesia about 17,000 years ago ${ }^{29,30}$ and of the Denisova mtDNA lineage in southern Siberia about 40,000 years ago suggest that multiple Late Pleistocene hominin lineages coexisted for long periods of time in Eurasia.

Received 21 January; accepted 3 March 2010.

Published online 24 March; corrected 8 April 2010 (see full-text HTML version for details).

1. Briggs, A. W. et al. Targeted retrieval and analysis of five Neandertal mtDNA genomes. Science 325, 318-321 (2009).

2. Derevianko, A. P., Petrin, V. T. \& Rybin, E. P. The Kara-Bom site and the characteristics of the Middle to Upper Palaeolithic transition in the Altai. Archaeol. Ethnol. Anthropol. Eurasia 2, 33-52 (2000).

3. Goebel, T., Derevianko, A. P. \& Petrin, V. T. Dating the Middle-to-UpperPaleolithic Transition at Kara-Bom. Curr. Anthropol. 34, 452-458 (1993).

4. Krause, J. et al. Neanderthals incentral Asia and Siberia. Nature 449, 902-904 (2007). each major node. The map shows the geographical origin of the mtDNAs $(24,25,32,44$ are in the Americas). Note that two partial mtDNAs sequenced from Teshik Tash and Okladikov Cave in Central Asia fall together with the complete Neanderthal mtDNAs in phylogenies ${ }^{4}$ (not shown).
5. Gabunia, L., Vekua, A. \& Lordkipanidze, D. The environmental contexts of early human occupation of Georgia (Transcaucasia). J. Hum. Evol. 38, 785-802 (2000).

6. Hublin, J. J. The origin of Neandertals. Proc. Natl Acad. Sci. USA 106, 16022-16027 (2009).

7. Noonan, J. P. et al. Sequencing and analysis of Neanderthal genomic DNA. Science 314, 1113-1118 (2006).

8. Stringer, C. B. \& Hublin, J. New age estimates for the Swanscombe hominid, and their significance for human evolution. J. Hum. Evol. 37, 873-877 (1999).

9. Schoetensack, O. Der Unterkiefer des Homo heidelbergensis aus den Sanden von Mauer bei Heidelberg (Engelmann, 1908).

10. Tattersall, I. Out of Africa: modern human origins special feature: human origins: out of Africa. Proc. Natl Acad. Sci. USA 106, 16018-16021 (2009).

11. Stringer, C. New views on the origin of our species. R. Soc. Arts J. 145, 111-116 (1997).

12. Caramelli, D. et al. A 28,000 years old Cro-Magnon mtDNA sequence differs from all potentially contaminating modern sequences. PLoS One 3, e2700 (2008).

13. Krause, J. et al. A complete mtDNA genome of an early modern human from Kostenki, Russia. Curr. Biol. 20, 231-236 (2010)

14. Krings, M. et al. Neandertal DNA sequences and the origin of modern humans. Cell 90, 19-30 (1997).

15. Bermudez de Castro, J. M. et al. A hominid from the lower Pleistocene of Atapuerca, Spain: possible ancestor to Neandertals and modern humans. Science 276, 1392-1395 (1997)

16. Smith, C. I., Chamberlain, A. T., Riley, M. S., Stringer, C. \& Collins, M. J. The thermal history of human fossils and the likelihood of successful DNA amplification. J. Hum. Evol. 45, 203-217 (2003).

17. Swisher, C. C. III et al. Latest Homo erectus of Java: potential contemporaneity with Homo sapiens in southeast Asia. Science 274, 1870-1874 (1996).

18. Bolikhovskaya, N. S., Derevianko, A. P. \& Shunkov, M. V. The fossil palynoflora, geological age, and climatostratigraphy of the earliest deposits of the Karama site (Early Paleolithic, Altai Mountains). Paleontol. J. 40, 558-566 (2006). 
19. Viola, T. B., Teschler-Nicola, M., Schaefer, K., Derevianko, A. \& Seidler, H. Postcranial remains from Okladnikov Cave, Siberia. Am. J. Phys. Anthropol. 46, 214-215 (2008).

20. Derevianko, A. P. in The Paleolithic of Siberia - New Discoveries and Interpretation (ed. Derevianko, A. P.) (Univ. Illinois Press, 1998).

21. Briggs, A. W. et al. Removal of deaminated cytosines and detection of in vivo methylation in ancient DNA. Nucleic Acids Res.. doi:10.1093/nar/gkp1163 (22 December 2009).

22. Kircher, M., Stenzel, U. \& Kelso, J. Improved base calling for the Illumina Genome Analyzer using machine learning strategies. Genome Biol. 10, R83 (2009).

23. Andrews, R. M. et al. Reanalysis and revision of the Cambridge reference sequence for human mitochondrial DNA. Nature Genet. 23, 147 (1999).

24. Briggs, A. W. et al. Patterns of damage in genomic DNA sequences from a Neandertal. Proc. Natl Acad. Sci. USA 104, 14616-14621 (2007).

25. Brotherton, P. et al. Novel high-resolution characterization of ancient DNA reveals $\mathrm{C}>\mathrm{U}$-type base modification events as the sole cause of post mortem miscoding lesions. Nucleic Acids Res. 35, 5717-5728 (2007).

26. Green, R. E. et al. A complete Neandertal mitochondrial genome sequence determined by high-throughput sequencing. Cell 134, 416-426 (2008).

27. Hublin, J. J. Out of Africa: modern human origins special feature: the origin of Neandertals. Proc. Natl Acad. Sci. USA 106, 16022-16027 (2009).

28. Derevianko, A., Shunkov, M. \& Volkov, P. A Paleolithic bracelet from Denisova cave. Archaeology. Ethnol. Anthropol. Eurasia 34, 13-25 (2008).
29. Brown, P. et al. A new small-bodied hominin from the Late Pleistocene of Flores, Indonesia. Nature 431, 1055-1061 (2004).

30. Morwood, M. J. et al. Archaeology and age of a new hominin from Flores in eastern Indonesia. Nature 431, 1087-1091 (2004).

Supplementary Information is linked to the online version of the paper at www.nature.com/nature.

Acknowledgements We thank A. W. Briggs, K. Finstermeier, R. E. Green, J.-J. Hublin, M. Kircher, A. I. Krivoshapkin, T. Maricic, M. Stoneking, A. Tsybonkov, L. Vigilant and V. Wiebe for comments, M. Meyer, the MPI-EVA sequencing group and $M$. Kircher for technical support, and the Presidential Innovation Fund of the Max Planck Society for financial support. J.M.G. was supported during this research by an NSF international postdoctoral fellowship (OISE-0754461).

Author Contributions Q.F. and J.K. performed the experiments; J.K., J.M.G. and Q.F. analysed the data; B.V., M.V.S. and A.P.D. provided expert archaeological and anthropological information; J.K., J.M.G. and S.P. wrote the manuscript.

Author Information The sequence of the Denisova mtDNA is deposited at the EMBL nucleotide database under accession number FN673705. Reprints and permissions information is available at www.nature.com/reprints. The authors declare no competing financial interests. Correspondence and requests for materials should be addressed to J.K. (krause@eva.mpg.de). 